\title{
La influencia de Pistorius en la definición kantiana de la metafísica a partir de 1787
}

Pistorius' influence on Kant's definition of metaphysics since 1787

\author{
Gabriel Rivero \\ gabrielrivero78@gmail.com \\ (Universität Mannheim, Mannheim, Alemania)
}

Resumen: El presente artículo trata acerca del concepto de metafísica de Kant a partir de 1787, año en el cual se in troduce en la definición kantiana el tér ${ }^{-}$ mino suprasensible. El artículo parte dē la concepción, según la cual las críticas de Pistorius al sistema transcendental llevan a Kant a presentar una nueva definición de metafísica, que se caracteriza por la asimilación y resignificación del concepto suprasensible en términos de una filosofía crítica. Una comparación con los Progresos indicará que la definición kantiana de metafísica en los años noventa se ha de interpretar de acuerdo a la ya esbozada en 1787 .

Palabras clave: suprasensible; Crítica de la razón práctica; metafísica; sensibilidad; libertad
Abstract: The present article deals with Kant's concept of metaphysics from 1787 onwards, the year in which the term supersensible has been introduced to Kant's definition of metaphysics. The article assumes that Pistorius' criticism of the transcendental system caused Kant to present a new definition of metaphysics, which is characterized by an assimilation and reconception of the term supersensible in the sense of a critical philosophy. A comparison with the Progresses will prove that the Kantian definition of metaphysics in the 1790s must be interpreted according to the draft of 1787 .

Keywords: supersensible; Critique of practical reason; metaphysics; sensibility; freedom

DOI: http://dx.doi.org/10.11606/issn.2318-9800.v19i2p111-130

Si bien se la puede considerar decisiva, la influencia de Hermann Andreas Pistorius en el pensamiento kantiano no ha sido aún investigada exhaustivamente. ${ }^{1}$ En lo que sigue se mostrará que uno de los

1. Las recientes investigaciones de Bernward Gesang und Bernd Ludwig abren camino, sin embargo, a cambiar esta situación.Véase GESANG, B. Kants 
múltiples aspectos que da cuenta de la relevancia de las reseñas de Pistorius es la posible influencia en la definición kantiana de la metafísica a partir de 1787. La influencia de Pistorius no ha de restringirse entonces, como bien podría pensarse, a cuestiones específicas de la filosofía práctica, sino más bien ha de ampliarse a la totalidad del sistema kantiano. Tal apreciación de la influencia de las críticas al sistema kantiano se dejan apreciar especialmente en la reseña al libro de Schultz Erläuterungen des Herrn Prof. Kants Critik der reinen Vernunft, que apareciera en el año 1784 y que Pistorius reseñara hacia 1786. Partiendo de esta reseña se ha de mostrar que las críticas esbozadas por el autor se manifiestan luego en cambios decisivos a partir de 1787, cuyas principales consecuencias se consolidan en la introducción del concepto de lo suprasensible (Übersinnliche) y la de ahí resultante nueva definición de metafísica. En tanto la metafísica se define en relación a lo suprasensible, resulta claro que su relevancia se extiende hasta los años noventa, en los cuales la metafísica se determina siguiendo tal concepto, como es el caso de los Progresos de la metafísica.

En lo que sigue se presentarán los siguientes aspectos: El punto I esboza algunas definiciones de la metafísica en el transcurso del desarrollo del pensamiento kantiano. Con ello se pretende situar la definición de la edición-B de la primera Crítica en el contexto del pensamiento kantiano y mostrar sus diferencias respecto de las fases anteriores. El punto II trata la cuestión del surgimiento del concepto de lo suprasensible desde 1786 y su conexión con la Crítica de la razón práctica. Por último, el punto III ofrece una interpretación de la definición de la metafísica de los Progresos a la luz de los puntos I y II. Como conclusión del análisis desarrollado en los tres puntos se pretende mostrar que la definición de la metafísica de los años noventa,

vergessener Rezensent: Die Kritik der theoretischen und praktischen Pbilosopbie Kants in fünf Rezensionen von Hermann Andreas Pistorius. Hamburg: Felix Meiner, 2007. LUDWIG, B. Die ,consequente Denkungsart der speculativen Kritik'. Kants radikale Umgestaltung seiner Freiheitslehre im Jahre 1786 und die Folgen für die kritische Philosophie als Ganze. En: Deutsche Zeitschrift für Pbilosopbie, 58, 2010, pp. 595-628. Del mismo autor: Kants Bruch mit der schulphilosophischen Freiheitslehre im Jahre 1786 und die ,Consequente Denkungsart der speculativen Kritik'. En: BACIN, S., FERRARIN, A., LA ROCCA, C., RUFFING, M. (ed.) Kant und die Pbilosopbie in weltbïrgerlicher Absicbt. Akten des XI. Internationalen Kant-Kongresses. Berlin/Boston: Walter de Gruyter, 2013, pp. 371-384. 
que a todas luces parece ser nueva, se ha de interpretar en conexión con la crítica de Pistorius, la segunda edición de la Crítica de la razón pura y la Crítica de la razón práctica.

\section{Definiciones de la metafísica hasta 1781}

Si se consideran las definiciones de la metafísica hasta 1781, puede decirse que hasta esa fecha se destacan en general tres grandes etapas del pensamiento kantiano: a) $1762 / 1764$, b) $1769 / 1770$ y c) 1781.

El primero de los períodos mencionados se caracteriza por una definición de la metafísica que se encuentra muy próxima a la de Baumgarten. Kant define en ese momento a la metafísica del siguiente modo: "La metafisica no es otra cosa que una filosofía sobre los primeros fundamentos de nuestro conocimiento."2 Por su parte, Baumgarten presenta en el $\S 1$ de la Metaphysica la definición que sigue: "Ciencia de los primeros principios del conocimiento humano."3 Por medio de una comparación entre las definiciones citadas se hace evidente que el punto crítico de Kant respecto a la metafísica tradicional ha de verse hacia 1762 únicamente en la introducción de un nuevo método, a saber el analítico. Las convicciones de Kant en lo que atañe a la posibilidad del conocimiento en cuestiones metafísicas restan hasta ese entonces intocadas, mientras que su definición de metafísica guarda, en general, una amplia similitud con la de Baumgarten.

Más bien es el segundo de los períodos arriba mencionados que trae consigo una definición de la metafísica en un sentido kantiano más propio. Las Reflexiones de la fase $\kappa$ de 1769 continúan y radicalizan la concepción de 1766. En los Sueños de un visionario Kant elabora una nueva definición, en la cual la metafísica se determina como conoci-

2. UD, AA II, p. 283. Las citas de las obras de Kant se harán siguiendo la sigla alemana de la obra, referencia a la "Akademie Ausgabe" (AA), número romano del volumen correspondiente y por último el número de página. Las obras se citan de acuerdo a KANT, I. Gesammelte Scbriften, Preußische Akademie der Wissenschaften. Berlin: De Gruyter, 1900. Una lista de las siglas utilizadas se encuentra al final del artículo. Las traducciones de los textos son responsabilidad del autor.

3. BAUMGARTEN, A. Metapbysica/Metapbysik, edición crítica, introducción y traducción de Günter Gawlick/Lothar Kreimendahl. Stuttgart Bad-Cannstatt: Frommann-Holzboog, 2011, §1. 
miento de los "límites de la razón humana" (Grenze der menschliche Vernunft). ${ }^{4} 1766$ trae consigo entonces un cambio en el pensamiento kantiano, en tanto el conocimiento se define a partir de los límites de la razón. 1769 continúa esta concepción; sin embargo, se introduce en ese año un elemento nuevo que sirve como punto de partida para la radicalización de las diferencias de Kant respecto de la tradición racionalista, como así también de Kant mismo respecto de sus concepciones de los años anteriores. La fase $\kappa$ no sólo destaca la idea de los límites de la razón, sino también aquella del "origen" (Ursprung) de los conceptos. A partir de allí, Kant utiliza este elemento, por un lado, para diferenciar la lógica de la metafísica, y, por otro, para criticar de manera radical la concepción baumgartiana. A diferencia de la metafísica, la lógica trata de la "subordinación" (Unterordnung) de las representaciones, sin determinar ningún tipo de restricción respecto a su origen. La amplitud de la definición de Baumgarten tiene como consecuencia la imposibilidad de determinar los límites de una y otra ciencia, de manera tal, que los límites y definición de la metafísica no pueden ser comprendidos con precisión.

La metafísica accede al conocimiento solo por medio de la razón, la lógica trata de todo [conocimiento]. Todos los conceptos de la razón son generales; la lógica muestra la relación de lo general con lo particular. Pero la metafísica [muestra] el origen de los conceptos generales, hacia los cuales todo conocimiento debe ser reconducido. ${ }^{5}$

Esta Reflexión indica entonces que el criterio para determinar el conocimiento específico de la metafísica se ha de llevar a cabo desde una doble perspectiva: por un lado, a través de la limitación del conocimiento al conocimiento racional y puro; por el otro, a través de la determinación del origen de los conceptos. Por medio del primer punto mencionado se limita la metafísica a un ámbito en el cual nada empírico ha de tomarse en consideración, en tanto el conocimiento solo se basa en la razón. A través del segundo elemento mencionado se sigue, por tanto, una nueva concepción de la metafísica, que Kant

4. TG, AA II, p. 368.

5. Refl 3946, AA XVII, p. 359. "Die logic läßt die besondere Natur der Menschlichen Vernunft unbestimmt und gilt vor jeder Vernunft ${ }_{i}$ die metaphysik zeigt die allgemeinen Begriffe, die aus der Natur der menschlichen Vernunft fließen, und deren besondere Gesetze." Refl 3946, AA XVII, p. 360. 
en un gran número de Reflexiones acentúa, a saber, la determinación subjetiva y crítica del origen del conocimiento. ${ }^{6}$ Una investigación sobre el origen de nuestros conceptos lleva entonces necesariamente a una investigación sobre nuestra razón bumana. De aquí se sigue la siguiente definición de la fase $\kappa$ : "La metafísicia es [...] una ciencia de los conceptos y principios de la razón humana y no del conocimiento humano en general, en el cual se incluye lo empírico y lo sensible." ${ }^{17}$

Con lo dicho anteriormente se arriba por primera vez a una verdadera delimitación y posición crítica respecto a la definición de Baumgarten, cuya importancia se extiende hasta el período más maduro de Kant. Ella se basa en la introducción de la razón bumana (Kant) en contraposición al conocimiento bumano (Baumgarten) como objeto de la metafísica. ${ }^{8}$ De ese modo, la metafísica, como ciencia separada de la sensibilidad, se ocupa solamente de los conceptos puros de la razón humana y sus límites. A diferencia del período 1762/1764 resulta entonces en 1769 una definición que combina los siguientes elementos: los límites, la razón humana y lo subjetivo. ${ }^{9}$

En lo que sigue se presenta la tercera de las etapas mencionadas, es decir, 1781. En el "Prefacio" de la edición-A de la primera Crítica se

6. Compárese entre otras las Refl. 3939, 3948, 3950 y 3952.

7. Refl 3946, AA XVII, p. 359.

8. La misma crítica a Baumgarten se encuentra en la Vorlesung über die Metapbysik $L_{1}$ : "Man hat niemals recht gewußt, was Metaphysik sey, obgleich sie so lange ist tractirt worden. Man wußte ihre Grenzen nicht zu bestimmen; daher setzte man vieles herein, was nicht darein gehörte; welches auf der Definition beruhte, indem man sie durch die ersten Principien der menschlichen Erkenntniß definirte." V-Met-L1/Pölitz, AA XXVIII, pp. 172 y 223. Véase también la Metapbysik Volckmann, en la cual Kant reproduce la misma crítica: V-Met/Volckmann, AA XXVIII, p. 367.

9. De tal restricción a los conceptos puros de la razón resulta otra determinación esencial para el conocimiento metafísico: la distinción entre filosofía subjetiva y objetiva. La Reflexión 3952 da cuenta de ello cuando Kant escribe: "Die Metaphysik ist eine Wissenschaft von den Gesetzen der reinen Menschlichen Vernunft und also subiectiv. Die obiective reine philosophie hat entweder analytische principia ohne alle Erfahrungsaxiomen oder synthetische. Die erstere beruhet auf allgemeinen Urtheilen nach der Regel der identitaet und auf der unterordnung des besonderen unter das allgemeine [...] Die zweyte hat zum obiect das allgemeinste des äusseren und inneren Sinnes." Refl 3952, AA XVII, pp. 362-363. 
encuentra la conocida concepción de la metafísica como aquella ciencia que se confunde irremediablemente en contradicciones. La razón, llevada por su necesidad arquitectónica de conocer más allá de la experiencia, arriba a un saber que no es más que el "lugar de infinitas disputas. ${ }^{10}$ En contraposición a la definición negativa del "Prefacio" el capítulo sobre la "Arquitectónica de la razón pura" ofrece, sin embargo, una definición positiva. En este lugar la metafísica es concebida como la ciencia que trata del conocimiento racional por "meros conceptos" (bloße Begriffe) y cuyos objetos de conocimiento son la naturaleza y la libertad. Siendo naturaleza y libertad objeto de la metafísica, ella refiere esencialmente a los "fines" (Zwecke) de la razón humana y como tal orienta el sistema del conocimiento hacia ellos. De ese modo, la metafísica es la "completitud de toda cultura de la razón humana, en tanto toma en consideración las finalidades y las máximas más altas de todo conocimiento humano." ${ }^{11}$ La metafísica tiene entonces una orientación práctica, que se manifiesta en el "concepto cósmico" (Weltbegriff), que Kant contrapone al "concepto escolástico" (Schulbegriff). Kant retoma con ello una crítica ya desarrollada a partir de los inicios de los años setenta, en los cuales Baumgarten y Wolff son criticados por su concepción de la arquitectónica del sistema y, correspondientemente, por su falta de orientación práctica en la determinación del conocimiento en general. ${ }^{12}$

\section{La cuestión de lo suprasensible a partir de 1787}

A través de lo expuesto anteriormente se deja reconocer con facilidad que hasta 1781 no se encuentra ningún pasaje en el corpus de la obra kantiana, en el cual se mencione explícitamente el concepto de lo suprasensible en relación con el concepto de metafísica. A primera vista, parece ser el escrito sobre los Progresos de la metafísica el primero que recién pone de manifiesto tal relación. ${ }^{13}$ Cabe entonces

10. $\mathrm{KrV}$ A VIII.

11. $\mathrm{KrV}$ A $851 / \mathrm{B} 879$.

12. Véase HINSKE, N. Zwischen Aufklärung und Vernunftkritik. Studien zum Kantschen Logikcorpus. Stuttgart-Bad Cannstatt: Frommann-Holzboog, 1998.

13. "[E]1la [scil. metafísica] es la ciencia de progresar del conocimiento de lo sensible a lo suprasensible a través de la razón." FM, AA XX, p. 260. Acerca del origen y datación del texto de los Progresos véase MOHR, G. Kants Grundlegung der kritischen Pbilosopbie. Werkkommentar und Stellenkommentar zur Kritik 
preguntarse si acaso en los años noventa se ocupaba Kant de una fundamentación de la metafísica general y especial (Ontología, Cosmología, Psicología y Teología) ${ }^{14}$ y si cambia de esta manera la concepción de 1781 con el recurso a lo suprasensible. Sin embargo, ella aparece claramente en la edición-B de la primera Crítica, como así también en la Crítica de la razón práctica. ¿Por qué razones Kant define a partir de 1787 la metafísica en relación con lo suprasensible?

Una respuesta a esta cuestión puede darse siguiendo el desarrollo histórico del concepto de lo suprasensible en Kant. Con ello puede mostrarse que lo suprasensible no ha de entenderse de ningún modo como una novedad de los Progresos ni tampoco de la Crítica del juicio. Más bien, se hace claro que tal concepto aparece mucho antes y justamente en conexión con problemas que se originan ya la primera Crítica de 1781 y a los cuales la Crítica de la razón práctica intenta dar una respuesta.

La introducción del concepto de lo suprasensible se lleva a cabo desde dos grupos de textos particulares. En el primero, lo suprasensible aparece como un aspecto crítico hacia las teorías de la metafísica tradicional $l_{i}$ en el segundo, por el contrario, el concepto experimenta una asimilación y resignificación en el sistema kantiano, asignándole un sentido positivo. Dicho de otro modo, el concepto de lo suprasensible surge, por un lado, como punto de partida crítico a las teorías tradicionales y, por el otro, como instancia de precisión del sistema transcendental. En 1787 se experimenta, por lo tanto, un cambio en la concepción kantiana, en tanto se reasume y resignifica el concepto tradicional de lo suprasensible, ofreciendo la incorporación del mismo al sistema transcendental.

El primero de ambos grupos de textos mencionados data del año 1786. En este sentido, es de destacar la obra de Jakob Prïfung der

der reinen Vernunft, zu den Prolegomena und zu den Fortschritten der Metaphysik. Frankfurt am Main: Suhrkamp, 2004, pp. 557 y ss; como así también VLEESCHAUWER, H. DE La Composition du Preisschrift d'Immanuel Kant sur les progrès de la métaphysique. En: Journal of the bistory of philosopby, 17, 1979, pp. 143-196.

14. Véase CAIMI, M. Kants Metaphysik. Zu Kants Entwurf einer metaphysica specialis. En: FUNKE, G. (ed.) Akten des siebten Internationalen Kant-Kongresses. Bonn-Berlin: Bouvier Verlag, 1991, pp. 103-126. Del mismo autor: La metafísica de Kant. Reconstrucción de la argumentación del escrito de Kant "Los progresos de la metafísica desde la época de Leibniz y de Wolff." Buenos Aires: Eudeba, 1989. 
Mendelssonschen Morgenstunden. En consonancia con su crítica a Mendelssohn, Jakob tematiza el adjetivo "suprasensible" (übersinnlich) con un tono crítico. ${ }^{15}$ Lo mismo puede decirse de Kant, que en sus Bemerkungen al texto de Jakob aplica el concepto de lo suprasensible en un sentido idéntico. De tal modo, se hace evidente que Kant se refiere a lo suprasensible para expresar una crítica al intento de extender el conocimiento sobre los límites de la experiencia. ${ }^{16}$ Lo suprasensible se restringe más bien a un aspecto negativo del término y puede, por tanto, contraponerse al sistema transcendental. Otro pasaje, en el cual Kant parte de la misma intención crítica, se encuentra el escrito ¿Qué significa orientarse en el pensamiento? de 1786. Kant toma partido en este escrito de la discusión entre Mendelssohn y Jacobi en torno al panteísmo y en conexión con ello critica nuevamente a Mendelssohn por su tratamiento del concepto de suprasensible. Consecuentemente, y en contraposición a él, Kant parte de la idea que orientarse en el pensamiento se corresponde con una necesidad subjetiva de la razón, por medio de la cual objetos suprasensibles solo pueden ser pensados. La razón reconoce con ello la necesidad de tales objetos, pero al mismo tiempo restringe tal necesidad a un fundamento subjetivo. ${ }^{17}$ Esta necesidad se divide igualmente en un uso teorético y en un uso práctico. El último es entendido en el contexto de una creencia racional

15. "Es ist wahr, die Wissenschaft der übersinnlichen Gegenstände hat abgenommen. [...] Freilich zerfallen nun alle die schönen Demonstrationen a priori für das Dasein der einfachen Substanzen und andrer übersinnlichen Dinge." JAKOB, L. H. Prüfung der Mendelssobnschen Morgenstunden oder aller spekulativen Beweise für das Dasein Gottes, Aetas Kantiana. Brüssel, 1768, p. XX y XXV. Otros pasajes, en los cuales se menciona lo suprasensible, son los siguientes: pp. XXII, XXXI, XXXIV, XXXV, XLVI, 13, 16.

16. "[...] statt dessen, wenn diesem [scil. Dogmatismus] in Ansehung des Uebersinnlichen durch strenge Kritik die Flügel beschnitten werden, jener Glaube in einer praktisch-wohlgegründeten, theoretisch aber unwiderleglichen Vorausssetzung gesichert sein kann." KANT, I. Einige Bemerkungen von Herrn Professor Kant. En: JAKOB, L.H. Prüfung der Mendelssobnschen Morgenstunden, p. LI.

17. "Nun aber tritt das Recht des Bedürfnisses der Vernunft ein, als eines subjectiven Grundes etwas vorauszusetzen und anzunehmen, was sie durch objective Gründe zu wissen sich nicht anmaßen darf; und folglich sich im Denken, im Unermeßlichen und für uns mit dicker Nacht erfüllten Raume des Übersinnlichen, lediglich durch ihr eigenes Bedürfniß zu orientieren." WDO, AA VIII, p. 137. 
(Vernunftglauben), que se ha de determinar sólo como máxima de la razón en lo que respecta a los objetos de naturaleza suprasensible. A partir de estos conceptos, resulta la siguiente conclusión: "Mendelssohn no pensó que dogmatizar con la razón pura en el ámbito de lo suprasensible fuera el camino directo al entusiasmo (Schwärmerei) filosófico."18 Lo dicho anteriormente muestra entonces que el uso del concepto de los suprasensible hacia 1786 está conectado principalmente con un propósito polémico, fundamentalmente dirigido contra Mendelssohn e inspirado por Jakob. ${ }^{19}$

$\mathrm{Al}$ grupo de textos de 1786 le sigue un segundo del año 1787. Este último grupo toma una relevancia particular para la interpretación de lo suprasensible, porque es aquí donde se muestra un uso positivo del concepto, de manera tal que puede hablarse de una asimilación, pero bajo los límites del sistema transcendental. El primer texto relevante, en este sentido, es el "Prefacio" a la edición-B de la primera Crítica. Allí se presenta por primera vez una distinción - similar a la de los Progresos - entre lo sensible y lo suprasensibe. ${ }^{20}$ Particularmente importante en el contexto de este pasaje es, por un lado, la división de la metafísica en dos ámbitos, que correspondientemente son referidos a lo sensible y lo suprasensible; por otro, la conexión introduci-

18. Idem, ibidem.

19. En lo que respecta al análisis histórico del origen del concepto de lo suprasensible véase SCHWAIGER, C. Denken des, Übersinnlichen' bei Kant. Zu Herkunft und Verwendung einer Schlüsselkategorie seiner praktischen Metaphysik. En: FISCHER, N. (ed.) Kants Metapbysik und Religionspbilosopbie. Hamburg: Felix Meiner, 2004, pp. 331-345. Schwaiger defiende la tesis, según la cual la fuente del concepto kantiano se encuentra en los escritos de Johann August Eberhard. En su Antrittsvorlesung de 1778 define Eberhard la metafísica en relación con lo suprasensible del modo siguiente: "Diese Gleichartigkeit und wesentliche Verbindung der übersinnlichen und aussersinnlichen Wahrheiten macht es daher nicht allein bequem sondern auch nothwendig, daß beyde Arten in Eine Wissenschaft zusammengefaßt werden, und diese Wissenschaft ist die Metaphysik." EBERHARD, J. A. Von dem Begriffe der Pbilosopbie und ibren Theilen. Berlin, 1778, pp. 39-40. Asimismo recurre Eberhard al concepto de lo suprasensible - esta vez en forma de adjetivo (ïbersinnlich) - en su Vorbereitung zur natürlichen Theologie de 1781. Compárese EBERHARD, J.A. Vorbereitung zur natürlichen Theologie. Reprint en AA XVIII, en particular pp. 520 y 547.

20. Véase KrV B XXI-XXII. 
da entre el concepto de lo suprasensible y el conocimiento práctico: "Nos queda aún intentar, luego que a la razón especulativa le fuera negado avanzar en el campo de lo suprasensible, si la razón no encuentra data en su conocimiento práctico para determinar el concepto racional de lo incondicionado."21 Aquí se destaca con toda claridad que hacia 1787 Kant se confronta con dos nuevos aspectos de su filosofía, que hasta entonces no habían aparecido en toda su importancia: de una parte, lo suprasensible y de otra parte, el conocimiento práctico unido a él. ${ }^{22}$

¿Con qué propósito surgen estos nuevos aspectos? En primer lugar, podría considerarse que Kant presenta una estrategia argumentativa en vista de defenderse de algunas críticas. Tal estrategia consiste en una precisión y asimilación del concepto de lo suprasensible, que en un primer momento fuera criticado. Las precisiones, según la tesis defendida en el presente artículo, han de interpretarse como una reacción contra las objeciones de Pistorius. La reseña de Pistorius al libro de Schultz contiene algunos puntos críticos, a partir de los cuales el autor arriba a la importante conclusión, según la cual el sistema de la filosofía crítica resulta necesariamente inconsecuente, ya que sus mismos principios encierran contradicciones. La inconsecuencia inevitable del sistema kantiano tiene su origen en la relación establecida entre mundo sensible y mundo inteligible. La falsa relación entre mundo sensible y mundo inteligible se establece toda vez que Kant define espacio y tiempo como condiciones subjetivas del conocimiento. Partiendo de esa concepción de espacio y tiempo se arriba a falsas doctrinas, según Pistorius, que particularmente se muestran en el concepto de libertad. Como se puede apreciar, la críticas esbozadas se extiende hacia aquellos principios que Kant considera base de su filosofía transcendental, esto es, la doctrina del espacio y el tiempo y el concepto de libertad.

Si se plantea la cuestión de la relación entre mundo sensible y mundo inteligible (o bien entre fenómeno y objeto real), entonces resultan, de acuerdo a Pistorius, dos posibilidades para entenderla: tal

21. $\mathrm{KrV}$ B XXI.

22. Tal conexión puede ya verse en el capítulo del "Canon de la razón pura" de la edición-A de la primera Crítica. A pesar de ello, Kant no explicita aún de modo directo el concepto de lo suprasensible y de la metafísica en un sentido práctico como será entendido este último a partir de 1787. 
relación es subjetiva o bien objetiva. ${ }^{23}$ Considerando la primera posibilidad se llega a un resultado escéptico respecto al conocimiento en general de la metafísica. Solo cuando se acepta una relación objetiva, es posible garantizar un conocimiento metafísico de la realidad. Al respecto exige Pistorius que la concepción kantiana del espacio y del tiempo como intuiciones puras y condiciones subjetivas del conocimiento sea rechazada, pues sobre la base de ella se hace imposible una relación objetiva. En lugar de la concepción kantiana, espacio y tiempo han de comprenderse como "conceptos intermedios" (Mittelbegriffe), en los cuales una suerte de mediación entre lo sensible y lo suprasensible sea posible. Bajo tal comprensión del espacio y del tiempo resulta cognoscible la realidad de objetos suprasensibles. A tal efecto, Pistorius recurre al ejemplo del conocimiento del alma bajo los principios del espacio y el tiempo como conceptos intermedios:

La existencia real de un mundo inteligible y objetivo no sería más problemático, sino confiable y cierto. Aún más importante e interesante para nosotros es que nosotros no somos solo lógica y aparentemente, sino realmente sujetos individuales y pensantes, o substancias. $^{24}$

Si se entienden, por el contrario, espacio y tiempo como Kant lo hace en la primera Crítica, entonces resulta necesariamente la inconsecuencia del sistema. Según Pistorius, ello se muestra con particular evidencia en el concepto de libertad. La solución de Kant de la tercera antinomia, según la cual tanto la tesis como la antitesis han de aceptarse como válidas, de modo tal que pueda afirmarse tanto la determinación causal en los fenómenos como así también la existencia de una instancia independiente y espontánea (noumeno), es, según la perspectiva crítica de Pistorius, absolutamente contradictoria. Para aplicar un concepto consistente de la libertad Kant debería hacer un uso suprasensible de las categorías de causa-efecto y de substancia. De tal manera, Kant mismo debería superar el propio principio crítico de los límites de la experiencia. El uso transcendente de la categoría de causalidad resulta necesario, según Pistorius, toda vez que la libertad

23. PISTORIUS, H.A. Rezension von J. Schultzes Erläuterungen des Herrn Prof. Kants Critik der reinen Vernunft. En: LANDAU, A. (ed.) Rezensionen zur Kantischen Pbilosopbie 1781-1787. Bebra, 1991, pp. 331-332.

24. Idem, p. 339. 
debe ser efectiva en el mundo sensible, mientras que en el caso de la categoría de la substancia el uso transcendente resulta igualmente necesario porque se debe aceptar la existencia de un noumeno que actúe de modo espontáneo. ${ }^{25}$ La reseña pone, entonces, en primer plano tres problemas fundamentales de la filosofía kantiana, a saber: el problema ontológico de la mediación entre lo sensible y lo suprasensible, la crítica a la concepción subjetiva del espacio y el tiempo, y el cuestionamiento al acceso del conocimiento a la realidad de objetos suprasensibles.

La Crítica de la razón práctica, publicada en diciembre de $1787^{26}$, será entonces vista como la llave para las posibles soluciones a tales críticas. En este sentido, en una carta a Jakob de 1787 Kant afirma lo siguiente: "Ahora se encuentra mi Crítica de la razón práctica en de Grunert. Ella contiene algunos puntos que pueden superar los malentendidos de la crítica de la razón teórica." ${ }^{27}$ Si la segunda Crítica debe despejar los malentendidos surgidos de la primera, ellos no pueden haberse originado de las objeciones inmediatamente recibidas tras la publicación de la Crítica de la razón pura en 1781. A las críticas de FederGarve, según las cuales el idealismo transcendental no es más que un idealismo psicológico y escéptico, responde Kant con los Prolegomenos en $1783 .{ }^{28}$ Los malentendidos, de los que Kant habla en la carta de 1787 a Jakob, deben tener un carácter reciente y poco que ver con las críticas inmediatas tras la publicación de la primera Crítica en 1781. Si se pregunta cuáles pueden ser los nuevos malentendidos a los que Kant se refiere, una posible respuesta consiste en afirmar que las objeciones de Pistorius sobre la idealidad del espacio y la realidad de la libertad

25. Véase GESANG, B. Kants vergessener Rezensent: Die Kritik der theoretischen und praktischen Pbilosopbie Kants in fünf Rezensionen von Hermann Andreas Pistorius. Hamburg: Felix Meiner, 2007, p. XXXVIII.

26. Véase KLEMME, H. F. The origin and aim of Kant's Critique of practical Reason. En: REATH, A/TIMMERMANN, J. (ed.) Kant's Critique of practical Reason. A critical Guide. Cambridge: Cambridge University Press 2010, pp. 11-30.

27. Br, AA X, p. 494. Igualmente se expresa Kant en la segunda Crítica: "Nur eine ausführliche Kritik der praktischen Vernunft kann alle diese Mißdeutung heben." KpV, AA V, p. 6-7.

28. Véase Prol, AA IV, en particular Anmerkungen I-III y Anhang. Cómparese también la reseña de FEDER, J.G.H./GARVE, C. Rezension zur Kritik der reinen Vernunft. En: LANDAU, A. (ed.) Rezensionen zur Kantischen Pbilosopbie 1781-1787. Bebra, 1991, pp. 10-17. 
como causa inteligible representan las nuevas críticas a las que la filosofía transcendental se ve desafiada. La Crítica de la razón práctica puede evitar entonces este tipo de malentendidos, pues ella asegura el acceso al conocimiento de objetos suprasensibles en un sentido práctico y al mismo tiempo demuestra - de modo indirecto - que la concepción del espacio y del tiempo de la primera Crítica ha de juzgarse como correcta. Así, resulta una respuesta a los reparos de Pistorius. Dicho de otro modo, la Crítica de la razón práctica presenta la justificación de porqué el sistema crítico es consecuente. El "pensamiento consecuente" debe poder demostrar que el principio crítico de la limitación de la validez de las categorías con relación a la experiencia, cuyo elemento fundamental se encuentra en el teoría del espacio y el tiempo, es compatible con una causa inteligible y espontánea, es decir, con la posibilidad de la libertad. Tal justificación tiene lugar en la segunda Crítica a través de la introducción del conocimiento práctico, por un lado, y del factum de la razón, por el otro. ${ }^{29}$ Lo específico de esta nueva concepción muestra que la causalidad de la libertad se reconoce únicamente por medio de la ley moral como un hecho sin presupuestos. ${ }^{30}$ Siendo así, corresponde a la categoría de la causalidad una

29. La concepción del factum de la razón de 1787, de acuerdo a la interpretación de Ludwig, marca un cambio decisivo respecto del concepto de libertad, en tanto la primera Crítica como así también la Fundamentación de la metafísica de las costumbres se hallaban restringidas a un concepto teorético de la libertad, que recién en 1786 será cambiado a un concepto práctico. "Die Frage nach der Möglichkeit der Voraussetzung der Freiheit wird nämlich in beiden Schriften unmissverständlich als eine "speculative" bezeichnet und dementsprechend im Rahmen des Transzendentalen Idealismus jeweils mit Verweis auf Ideen beziehungsweise Apperzeption beantwortet. Dieser Teil der Freiheitsdeduktion findet 1781 wie 1785 also ausdrücklich auf dem Felde der theoretischen Philosophie statt - und genau das sollte sich 1786 ändern." LUDWIG, B. Die ,consequente Denkungsart der speculativen Kritik'. Kants radikale Umgestaltung seiner Freiheitslehre im Jahre 1786 und die Folgen für die kritische Philosophie als Ganze. En: Deutsche Zeitschrift für Pbilosopbie, 58, 2010, p. 609.

30. "Man kann das Bewußtsein dieses Grundgesetzes ein Factum der Vernunft nennen, weil man es nicht aus vorhergehenden Datis der Vernunft, z. B. dem Bewußtsein der Freiheit (denn dieses ist uns nicht vorher gegeben), herausvernünfteln kann, sondern weil es sich für sich selbst uns aufdringt als synthetischer Satz a priori, der auf keiner, weder reinen noch empirischen, Anschauung gegründet ist." KpV, AA V, p. 31. 
nueva determinación, en tanto ella, a través de la libertad (aus Freibeit), puede entenderse de modo objetivo y en un sentido práctico. Esto significa, que el acceso a la determinación de un objeto suprasensible de ningún modo presupone un conocimiento teórico, en tanto la libertad (según su posibilidad y realidad) es reconocida solo indirectamente a instancias de la ley moral (como ratio cognoscendi). Si se siguen estas directrices, se desprende de ello que ni la categoría de la causalidad ni la de substancia deben ser aplicadas teóricamente (y con ello de forma inconsecuente). Por tanto, la nueva doctrina del factum de la razón trae consigo una concepción de la libertad, mediante la cual se refutan todas las objeciones de Pistorius. ${ }^{31}$

Aquí se explica también por primera vez el acertijo de la Crítica, cómo se niega realidad objetiva al uso suprasensible de las categorías en la especulación y, sin embargo, se les concede esta realidad en vista de los objetos de la razón pura práctica. [ ] [R]azón práctica para sí misma [...] proporciona realidad a un objeto suprasensible de la categoría de causalidad, a saber a la libertad. ${ }^{32}$

La argumentación kantiana propone de esa manera que con el conocimiento práctico se puede hacer referencia libre de contradicciones a una causa inteligible. A través de la determinación de la voluntad, la cual por medio de la ley moral pone de manifiesto la causalidad de la libertad, no se arriba a un conocimiento teórico-dogmático, sino a una instancia práctica. ${ }^{33}$ Así, no se determinan objetos suprasensibles en un sentido real, sino que más bien se unifican en un único concepto con un sentido práctico, es decir, aquel del sumo bien (Höchstes Gut). ${ }^{34}$

31. Véase $\mathrm{KpV}, \mathrm{AA} \mathrm{V}$, p. 65-66.

32. Idem, p. 5-6.

33. "Freiheit ist aber auch die einzige unter allen Ideen der speculativen Vernunft, wovon wir die Möglichkeit a priori wissen, ohne sie doch einzusehen, weil sie die Bedingung des moralischen Gesetzes ist, welches wir wissen." $\mathrm{KpV}$, AA V, p. 4. "Die objektive Realität eines reinen Willens oder, welches einerlei ist, einer reinen praktischen Vernunft ist im moralischen Gesetz a priori gleichsam durch ein Factum gegeben [...] Im Begriffe eines Willens aber ist der Begriff der Causalität schon enthalten, mithin in dem eines reinen Willen der Begriff einer Causalität mit Freiheit." KpV, AA V, p. 55.

34. "Denn wir erkennen zwar dadurch weder unserer Seele Natur, noch die intelligibele Welt, noch das höchste Wesen nach dem, was sie an sich selbst 
La exigencia de Pistorius, según la cual espacio y tiempo deberían ser entendidos como conceptos intermedios, de tal forma que las dos dimensiones de un uso sensible y suprasensibles de las categorías ${ }^{35}$ se puedan conservar con sentido y sin contradicciones, son refutadas por Kant a través de una división aún más estricta, es decir, una división radical entre el uso teórico y práctico de la razón, y con ello la introducción de una resignificación de la distinción entre lo sensible y lo suprasensible como ámbitos sin mediación posible. De la separación de los campos de la metafísica (naturaleza y libertad), por un lado, y del conocimiento en un ámbito teórico y práctico, por otro, se desprende que la metafísica es una "tarea" (Aufgabe) de la razón, cuyo carácter esencial consiste fundamentalmente en el reconocimiento de la diferencia de sus principios constitutivos. En consonancia con las consideraciones de la primera y la segunda Crítica se llega así al resultado que la metafísica solo puede determinarse consecuentemente a partir de los presupuestos de la idealidad del espacio y del tiempo, por medio de los cuales se fundamenta el conocimiento teórico del mundo sensible, y de la realidad de la libertad (mundo inteligible), que se demuestra por el factum de la razón. ¿Cómo se debe interpretar, en relación con estos resultados, la concepción de la metafísica en los Progresos?

\section{Los Progresos de la metafísica a la luz de la Crítica de la razón práctica}

De lo expuesto anteriormente se puede concluir que, visto desde su desarrollo histórico, sería un error interpretar la introducción

sind, sondern haben nur die Begriffe von ihnen im praktischen Begriffe des höchsten Guts vereinigt, als dem Objecte unseres Willens, und völlig a priori durch reine Vernunft, aber nur vermittels des moralischen Gesetzes." $\mathrm{KpV}, \mathrm{AA} \mathrm{V}$, p. 133.

35. Un hecho que aún refuerza la presencia de la crítica de Pistorius se encuentra en la referencia de Kant a Spinoza. Kant señala en la segunda Crítica que de no aceptarse la teoría transcendental de espacio y tiempo, se llegaría al Spinozismo. En la reseña al libro de Schultz Pistorius acusa a Kant de acercarse a la posición de Spinoza. Una vez más se ve aquí una respuesta de Kant en la segunda Crítica a Pistorius. Véase PISTORIUS, H. A. Rezension von J. Schultzes Erläuterungen des Herrn Prof. Kants Critik der reinen Vernunft. En: LANDAU, A. (ed.) Rezensionen zur Kantischen Pbilosopbie 1781-1787. Bebra, 1991, p. 330. 
del concepto de lo suprasensible simplemente como una innovación de los Progresos de la metafísica. El hecho de que Kant ya en 1786 en el contexto de la polémica con Mendelssohn introduzca el concepto apoya de manera decisiva la posibilidad de interpretar el surgimiento de lo suprasensible como una reacción frente a las críticas a la filosofía trascendental. ${ }^{36}$ Por su parte, el segundo grupo de textos de 1787 confirma, entre otras cosas, que Kant se sirve del concepto de lo suprasensible en un sentido propio para defenderse, por un lado, de las objeciones y, a la vez, para introducir una precisión en su sistema.

De lo anterior se sigue que la definición de la metafísica en los Progresos representa una continuación de aquella de 1787. En primer lugar, se prosigue con el principio, según el cual la metafísica debe ser dividida en dos ámbitos. Si se acepta la tesis, según la cual Kant pretendió una metafísica trascendente a través de la innovación de la definición, se desconocería la agudeza de la separación de estos dos ámbitos del conocimiento, pues no se tomaría en cuenta, de ese modo, una de las particularidades fundamentales de la determinación de la filosofía transcendental que la diferencia de la tradición metafísica anterior, a saber, que respecto de la ontología no puede existir ninguna continuidad de principios que posibilite una transición de lo sensible a lo suprasensible: "[L]o suprasensible es distinto de lo cognoscible sensible, incluso según la especie (toto genere), porque se encuentra más allá de todo conocimiento posible. Por lo tanto, no existe ningún camino para llegar a él por los mismos progresos, a través de los cuales podemos esperar alcanzar certeza en el ámbito de lo sensible. ${ }^{1137} \mathrm{Si}$ no se estableciera tal división de principios, su continuidad llevaría a un regreso a la filosofía tradicional, que Kant justamente trata de criticar y poner sus límites. ${ }^{38}$ Una transición basada en una

36. Véase ÜE, AA VIII, pp. 195 y 216. Sobre el concepto de lo suprasensible véase MODEL, A. Zu Bedeutung und Ursprung von "Übersinnlich" bei Immanuel Kant. En: Archiv für Begriffsgeschichte, 30, 1986/87, pp. 183-191. Model señala los orígenes místicos del concepto y muestra de que manera Kant se distancia de tal entendimiento.

37. FM, AA XX, p. 299.

38. La correlación entre la idea de una continuidad de principios y la fundamentación de una metafísica en sentido tradicional es vista por Kant en la crítica de Eberhard a la filosofía trascencental:"Herr Eberhard sucht den Stoff zu aller Erkenntniß in den Sinnen, woran er auch nicht Unrecht thut. Er will aber doch auch diesen Stoff zum Erkenntniß des Übersinnlichen verarbeiten." 
continuidad de principios debe ser más bien ser entendida como salto (Überscbritt). ${ }^{39}$ Si te toma en cuenta esta pretensión de Kant en los Progresos, se hace insostenible la interpretación, según la cual en este escrito Kant retoma un modelo tradicional de la metafísica. ${ }^{40}$ Por ello, la metafísica debe ser definida desde dos ejes diferentes, que a su vez tomen en consideración la idealidad de la intuición y la realidad de la libertad. ${ }^{41}$ Con ello surge otro indicio que la concepción de 1787 coincide con la de 1793, esto es, que la metafísica se puede fundamentar y unificar por medio de la idealidad de la doctrina de espacio y tiempo (primera Crítica) y la realidad de libertad (segunda Crítica), sin que por ello se favorezca una transposición inconsecuente de sus principios, como Pistorius criticaba. Con la aparición de lo suprasensible como nuevo concepto de la metafísica no se produce, por lo tanto, un retorno a la tradición o un acercamiento a la concepción metafísica de Baumgarten. Esto ha pretendido demostrar la exposición de la

ÜE, AA VIII, p. 212. En cuanto a la importacia de Eberhard en el contexto de la redacción de los Progresos véase VLEESCHAUWER, H. DE op. cit., p. 145 .

39. "Die Transscendentalphilosophie, d.i. die Lehre von der Möglichkeit aller Erkenntniß a priori überhaupt, [ ] hat zu ihrem Zweck die Gründung einer Metaphysik, deren Zweck wiederum als Endzweck der reinen Vernunft, dieser ihre Erweiterung von der Grenze des Sinnlichen zum Felde des Übersinnlichen beabsichtiget, welches ein Überschritt ist, der, damit er nicht ein gefährlicher Sprung sey, indessen daß er doch auch nicht ein continuirlicher Fortgang in derselben Ordnung der Prinzipien ist, eine den Fortschritt hemmende Bedenklichkeit an der Grenze beyder Gebiete nothwendig macht." FM, AA XX, pp. 272-273.

40. Tal es el caso en la interpretación de Elena Ficara. "Das ganze metaphysische Gebiet - auch die Metaphysik als praktische Lehre - wird von Kant auf die Transzendentalphilosophie zurückgeführt, die als Begriffslehre interpretiert wird. Das bedeutet, dass die Transzendentalphilosophie Kants die Rolle der alten Ontologie als Grundlehre übernimmt." Véase FICARA, E. Die Ontologie in der "Kritik der reinen Vernunft". Würzburg: Königshausen \& Neumann, 2004, p. $132-133$.

41. "Das Übersinnliche, was gegeben ist, ist der Freyheitsbegriff; folglich ist kein synthetisch-theoretischer Satz, mithin der objectiv transscendent wäre, möglich." Refl. 6351, AA XVIII, p. 678. En esta misma Reflexión pregunta Kant por la posibilidad de juicios sintéticos a priori de lo suprasensible y la respuesta es la siguiente: "Als regulative Principien des Practischen." Ibidem. Véase también sobre el tema Refl. 6353, AA XVIII, p. 680-681. 
Gabriel Rivero

evolución histórica del concepto de lo suprasensible que se acaba de presentar.

\title{
Siglas
}

\author{
AA Akademie Ausgabe \\ Br Briefe \\ FM Welches sind die wirklichen Fortschritte, die die \\ Metaphysik seit Leibnizens und Wolff's Zeiten in \\ Deutschland gemacht hat? \\ KRV Kritik der reinen Vernunft \\ KPV Kritik der praktischen Vernunft \\ Refl Reflexionen \\ TG Träume eines Geistersehers, erläutert durch die \\ Träume der Metaphysik \\ V-Met-L/Pölitz Metaphysik L1 (Pölitz) \\ V-Met/Volckmann Metaphysik Volckmann \\ UD Untersuchung über die Deutlichkeit der \\ Grundsätze der natürlichen Theologie und der \\ Moral \\ ÜE Über eine Entdeckung, nach der alle neue Kritik \\ der reinen Vernunft durch eine ältere entbehrlich \\ gemacht werden soll \\ WDO Was heißt sich im Denken orientieren?
}

\section{Referências}

BAUMGARTEN, A. Metapbysica/Metapbysik. Edición crítica, introducción y traducción de Günter Gawlick/Lothar Kreimendahl. Stuttgart Bad-Cannstatt: Frommann-Holzboog, 2011.

CAIMI, M. La metafísica de Kant. Reconstrucción de la argumentación del escrito de Kant "Los progresos de la metafísica desde la época de Leibniz y de Wolff." Buenos Aires: Eudeba, 1989.

CAIMI, M. Kants Metaphysik. Zu Kants Entwurf einer metaphysica specialis. En: FUNKE, G. (ed.) Akten des siebten Internationalen KantKongresses. Bonn-Berlin: Bouvier Verlag, 1991, pp. 103-126.

EBERHARD, J. A. Von dem Begriffe der Pbilosopbie und ibren Theilen. Berlin, 1778.

EBERHARD, J.A. Vorbereitung zur natürlichen Theologie. Reprint en: AA XVIII. 
FEDER, J.G.H./GARVE, C. Rezension zur Kritik der reinen Vernunft. En: LANDAU, A. (ed.) Rezensionen zur Kantischen Pbilosophie 1781-1787. Bebra, 1991, pp. 10-17.

FICARA, E. Die Ontologie in der "Kritik der reinen Vernunft". Würzburg: Königshausen \& Neumann, 2004.

GESANG, B. Kants vergessener Rezensent: Die Kritik der theoretischen und praktischen Pbilosopbie Kants in fünf Rezensionen von Hermann Andreas Pistorius. Hamburg: Felix Meiner, 2007.

HINSKE, N. Zwischen Aufklärung und Vernunftkritik. Studien zum Kantschen Logikcorpus. Stuttgart-Bad Cannstatt: Frommann-Holzboog, 1998.

JAKOB, L. H. Prïfung der Mendelssobnschen Morgenstunden oder aller spekulativen Beweise für das Dasein Gottes, Aetas Kantiana. Brüssel, 1768.

KANT, I. Gesammelte Schriften, Preußische Akademie der Wissenschaften. Berlin: De Gruyter, 1900.

KLEMME, H. F. The origin and aim of Kant's Critique of practical Reason. En: REATH, A/TIMMERMANN, J. (ed.) Kant's Critique of practical Reason. A critical Guide. Cambridge: Cambridge University Press, 2010, pp. 11-30.

LUDWIG, B. Die, consequente Denkungsart der speculativen Kritik'. Kants radikale Umgestaltung seiner Freiheitslehre im Jahre 1786 und die Folgen für die kritische Philosophie als Ganze. En: Deutsche Zeitschrift für Pbilosopbie, 58, 2010, pp. 595-628.

LUDWIG, B. Bruch mit der schulphilosophischen Freiheitslehre im Jahre 1786 und die, Consequente Denkungsart der speculativen Kritik'. En: BACIN, S., FERRARIN, A., LA ROCCA, C., RUFFING, M. (ed.) Kant und die Pbilosopbie in weltbürgerlicher Absicht. Akten des XI. Internationalen Kant-Kongresses. Berlin/Boston: Walter de Gruyter, 2013, pp. 371-384.

MODEL, A. Zu Bedeutung und Ursprung von "Übersinnlich" bei Immanuel Kant. En: Archiv für Begriffsgeschichte, 30, 1986/87, pp. 183-191.

MOHR,G. Kants Grundlegung der kritischen Pbilosopbie. Werkkommentar und Stellenkommentar zur Kritik der reinen Vernunft, zu den Prolegomena und zu den Fortscbritten der Metaphysik. Frankfurt am Main: Suhrkamp, 2004.

PISTORIUS, H.A. Rezension von J. Schultzes Erläuterungen des Herrn Prof. Kants Critik der reinen Vernunft. En: LANDAU, A. (ed.) Rezensionen zur Kantischen Pbilosopbie 1781-1787. Bebra, 1991.

SCHWAIGER, C. Denken des, Übersinnlichen' bei Kant. Zu Herkunft und Verwendung einer Schlüsselkategorie seiner praktis- 
Gabriel Rivero

chen Metaphysik. En: FISCHER, N. (ed.) Kants Metapbysik und Religionspbilosopbie. Hamburg: Felix Meiner, 2004, pp. 331-345.

VLEESCHAUWER, H. DE La Composition du Preisschrift d'Immanuel Kant sur les progrès de la métaphysique. En: Journal of the bistory of philosopby, 17, 1979, pp. 143-196.

Recebido em 09.07.2014

Aceito em 22.10.2014 